\title{
Characterisation of Coherent Scatterers in Urban Areas by Means of Angular Diversity
}

\author{
Rafael Zandona Schneider, Konstantinos P. Papathanassiou, Irena Hajnsek and Alberto Moreira \\ Microwaves and Radar Institute \\ German Aerospace Center \\ PO BOX 1116, 82230, Wessling, Germany \\ Email: Rafael.Zschneider@dlr.de
}

\begin{abstract}
The detection of scatterers with pointwise response in SAR images of urban areas using the so-called Coherent Scatterers (CSs) technique was in previous works introduced [1], [2]. In this paper, the potential to exploit sub-aperture images from wide azimuth angular apertures (as it is the case for airborne systems) to estimate CSs backscattering radiation patterns is addressed. The backscattering radiation pattern is modeled assuming a simple canonical class of scatterers. The model is then inverted using the estimated radiation patterns of individual CSs and their geometrical parameters are evaluated and related to CSs polarimetric properties. Two experiments using dihedral corner reflectors with differentt azimuth orientation and Line of Sight (LOS) rotation angles were performed, in order to verify the agreement with the theory. The data used are at L-band from the airborne E-SAR system of the German Aerospace Center (DLR) and the testsite is the Munich city in Germany.
\end{abstract}

\section{INTRODUCTION}

The Coherent Scatterers (CSs) technique to detect scatterers with point-like behaviour in SAR images of urban areas was introduced in [1], [2]. Polarimetric and interferometric characterisation of CSs has been discussed, as well as the potential of physical and geometrical information extraction, as the estimation of the scatterers Line Of Sight (LOS) rotation angle and CSs dielectric properties.

In [3] the backscattering radiation pattern in the azimuth direction of the so-called Permanent Scatterers (PSs) was estimated using ERS-2 image series and taking advantage of its loss of the on-board gyroscopes. This allowed to acquire images of the same scene with different azimuth aspect angles. A backscattering pattern model for canonical PSs was used to invert PSs geometrical parameters.

In this work, azimuth backscattering radiation patterns of CSs are estimated using multi-look images from wide azimuth bandwidth systems. The backscattering radiation patterns are modeled as in [3] and the model parameters are inverted. Their relationship to the scatterer's type estimated through polarimetry is investigated. Finally, two experiments using dihedral corner reflectors were performed, in order to verify the agreement with the theory.

\section{CSs BACKSCATtering RAdiation Pattern Model}

Each scatterer in a radar-scatterer interaction reirradiates the incident electromagnetic energy, in a given frequency, according to its geometry and dielectric properties, among another factors. The backscattered energy varies in space

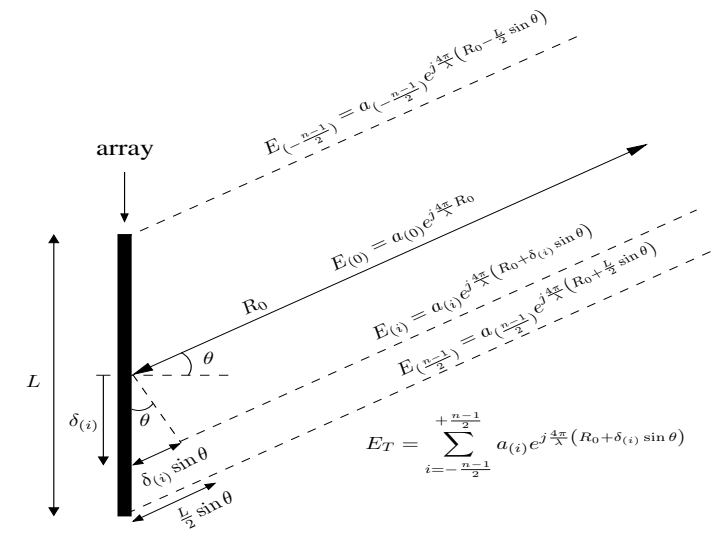

Fig. 1. Geometry of the backscattering of EM waves by a linear array of elements.

forming a pattern that is characteristic for each scatterer. In the case of a finite linear array of elements, for which the electromagnetic wavelength is much smaller than the array size but bigger than the individual elements (for a continuous object the elements are infinitesimal), the situation is that of Fig. 1.

The total backscattered field $E_{T}$ received far away from the scatterer (far field approximation) is the coherent sum of all individual fields $E_{(i)}$ backscattered from each element (Fig. 1). Assuming a continuous object of length $L$ constituted of infinitesimal elements that return constant amplitudes of values $A / L$, the coherent sum of the individual fields becomes

$$
E_{T}=\int_{-\frac{L}{2}}^{+\frac{L}{2}} \frac{A}{L} e^{j \frac{4 \pi}{\lambda} R_{0}} e^{j \frac{4 \pi}{\lambda} \delta \sin \theta} d \delta
$$

leading to

$E_{T}=A e^{j \frac{4 \pi}{\lambda} R_{0}} \operatorname{sinc}\left(\frac{2 L}{\lambda} \sin \theta\right)$, where $\operatorname{sinc}(x)=\frac{\sin (\pi x)}{\pi x}$

In order to allow a possible orientation $\theta_{S}$ of the scatterer in relation to the radar, and assuming that $\theta$ is small, (2) can be approximated by

$$
E_{T}=A e^{j \frac{4 \pi}{\lambda} R_{0}} \operatorname{sinc}\left[\frac{2 L}{\lambda}\left(\theta-\theta_{S}\right)\right]
$$

Certain CSs can be considered as such canonical objects and their backscattering radiation pattern can be modelled by 


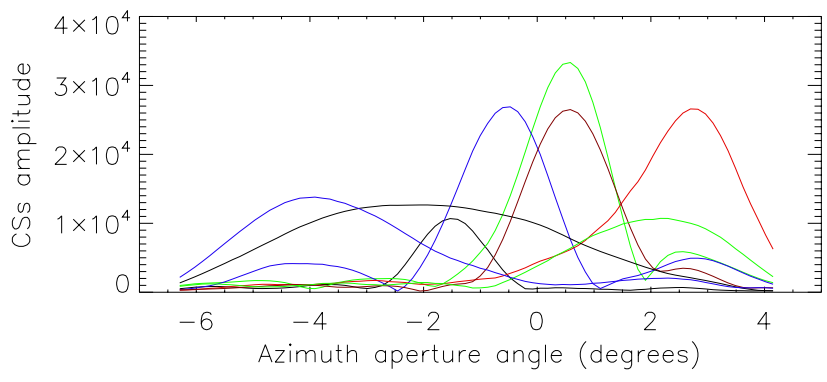

Fig. 2. CSs azimuth backscattering radiation pattern.

(3). Of course not all CSs will have such a simple radiation pattern, but as it will be shown in the following, a considerable number of CSs are in agreement with (3).

Equation (3) is general and can be applied to different directions. Azimuth aspect angle diversity can be achieved in SAR systems by evaluating different looks of the full azimuth spectrum, where each look corresponds to a different view of the imaged object. The diversity of aspect angles in the vertical direction can be obtained by multi-baseline acquisitions.

\section{CSS RADiATION PATTERN AND MODEL INVERSION}

In October 2005 the E-SAR system of the German Aerospace Center (DLR) flew over the city of Munich in Germany acquiring full polarimetric data at L-band. The processed images have an azimuth bandwidth of $179.6 \mathrm{~Hz}$ corresponding to an angular variation (azimuth aperture) of about $13.18^{\circ}$. The squint angle was about $-1.02^{\circ}$. A total of 89 sublook images were formed using a sub-bandwidth of $35.9 \mathrm{~Hz}(20 \%$ of the full bandwidth) and a step of $2.02 \mathrm{~Hz}$ along the whole available aperture $(0.80 * 179.6 \approx 143.6 \mathrm{~Hz})$. This corresponds approximately to an aspect angle variation of $10.54^{\circ}$. Fig. 2 shows some examples of estimated CSs radiation patterns in the azimuth direction for the $\mathrm{HH}$ polarization image. Note that the patterns are very similar to sinc functions. The patterns of the individual CSs have different widths and amplitudes of the main lobe and are centered at different positions in the aperture, indicating the presence of scatterers with different sizes and angular orientation. According to (3), the larger the main lobe the smaller the scatterer.

A fit of a sinc function according to (3) was performed in the azimuth radiation pattern for a representative number of the detected CSs. Fig. 3(a) shows the Root Mean Square (RMS) error in percentage of the sinc fit. One can see that in many cases a reasonable accurate fit was performed. CSs having a RMS error in the sinc fit less than $20 \%$ have been selected for further analysis. Figs. 3(b) to 3(d) show the histograms of the three estimated sinc parameters (CSs amplitude, azimuth size and orientation) for the selected CSs. The amplitude is very high, up to $40 \mathrm{~dB}$; the azimuth dimension varies between $70 \mathrm{~cm}$ to about $5 \mathrm{~m}$ with some higher concentration about $1.5 \mathrm{~m}$ and $3 \mathrm{~m}$; the azimuth orientation angle is diverse but has a concentration around the squint angle (scatterers directly faced to the radar LOS).

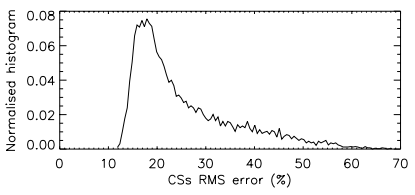

(a) RMS error in $\operatorname{sinc}$ fit.

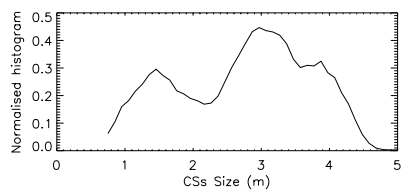

(c) Size of CSs with low RMS error.

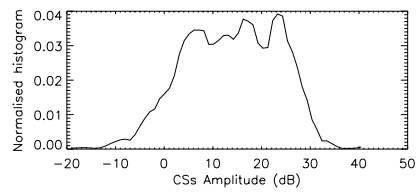

(b) Amplitude of CSs with low RMS error.

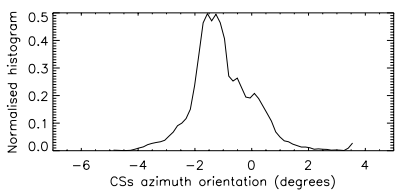

(d) Orientation of CSs with low RMS error.
Fig. 3. Inverted parameters' histograms of the CSs azimuth backscattering radiation pattern model.

\section{CSS POLARIMETRIC CHARACTERISTICS}

The polarimetric alpha angle $(\alpha)$ obtained from the coherence matrix decomposition [4] gives information about the type of the scattering mechanism occuring inside the resolution cell. For the case of deterministic scatterers as the CSs addressed here, $\alpha$ can be obtained directly from the scattering matrix as

$$
\alpha=\arccos \left(\frac{\left|S_{H H}+S_{V V}\right|}{\sqrt{\left|S_{H H}\right|^{2}+2\left|S_{H V}\right|^{2}+\left|S_{V V}\right|^{2}}}\right) .
$$

$\alpha$ ranges from $0^{\circ}$ to $90^{\circ}$. Low values of $\alpha\left(\leq 20^{\circ}\right)$ correspond to surface-like or trihedral scatterers while values around $45^{\circ}$ indicate dipole-like and high values $\left(\geq 60^{\circ}\right)$ dihedral scatterers. Fig. 4 shows the histogram of the polarimetric $\alpha$ angle for the CSs with the best sinc fits. By noticing the high concentration of dihedral, dipole, and surface-like scatterers in the histogram, one can suppose that these type of scatterers are the ones that best follow the model of (3).

Fig. 5 shows a set of two dimensional histograms relating the inverted sinc parametes, the polarimetric $\alpha$ angle, and the LOS rotation angle of CSs. Red indicates high while blue low number of occurrences.

Fig. 5(a) shows the relationship between amplitude and $\alpha$. All three types of scatterers have a wide range of amplitudes from $0 \mathrm{~dB}$ to about $35 \mathrm{~dB}$. Fig. 5(b) shows the histogram of the CSs size in relation to $\alpha$. One can see that the majority of the detected surface-like CSs have an azimuth dimension of about $3 \mathrm{~m}$ (they are in the class of big scatterers in Fig. 3(c)). On the other hand, dihedrals and dipoles are concentrated around the $1.5 \mathrm{~m}$ and $4 \mathrm{~m}$ azimuth dimension region lying on both classes of Fig. 3(c).

The relationship of the azimuth CSs orientation and $\alpha$ is given in Fig. 5(c). While dipoles and dihedrals are clustered around the squint angle (about $-1^{\circ}$ ), surface-like scatterers are more concentrated around $0^{\circ}$. 


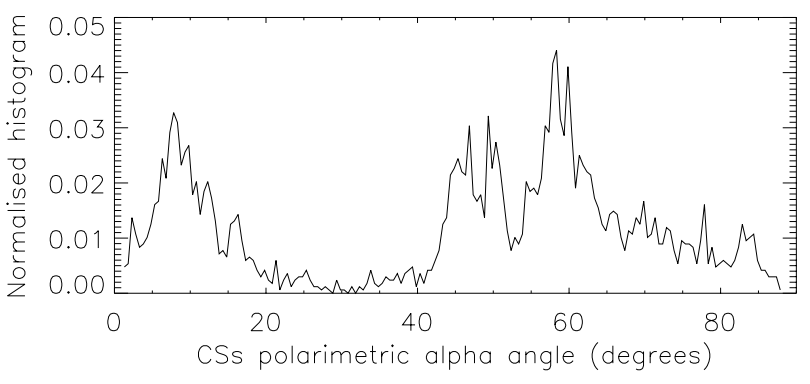

Fig. 4. CSs polarimetric $\alpha$ angle histogram.

The plot of the CSs amplitude versus size (Fig. 5(d)) shows a slight trend indicating that small scatterers have lower while large scatterers have higher amplitude. However, the opposite may ocasionaly happen (we have to emphasise that the vertical dimension of the scatterers is unknown, which influences the total backscattered amplitude). Note also the high concentration of $3 \mathrm{~m}$ CSs with high amplitude.

Fig. 5(e) shows that the two classes of CSs with dimensions around $1.5 \mathrm{~m}$ and $3 \mathrm{~m}$ have in general no preference related to their azimuth angular orientation. They have in general different orientations, however both classes slightly concentrate around the squint angle.

Finally, Fig. 5(f) presents the relationship of the azimuth orientation angle to the scatterers LOS rotation. The scatterer LOS rotation angle is estimated by exploiting the polarimetric signature of the individual CSs, as proposed in [5]. One can see that around the squint angle, a high concentration of CSs is present. However, scatterers with high LOS rotation and low angular orientation (an also the opposite situation) might be present in SAR images of urban areas. This means that both measurements are not totally dependent from eachother, leading to different/complementary information.

\section{DiHEDRAL CORNER REFleCtORS EXPERIMENTS}

In order to validate the estimated geometric parameters inverted by the sinc model, two experiments were performed using dihedral corner reflectors. In the first experiment two dihedrals were deployed parallel to the azimuth direction and faced to the radar LOS. One dihedral was vertically oriented while the other was rotated $5^{\circ}$ about the LOS. In the second experiment two other dihedrals were both deployed rotated at $45^{\circ}$ about the radar LOS but not aligned in azimuth: with $5^{\circ}$ difference in their azimuth orientation angle. Fig. 6 illustrates the difference of the LOS rotation $\phi$ and the azimuth angle orientation $\theta_{S}$. Note that the two effects are in general not independent from eachother. However, the LOS rotation of scatterers is mainly sensed by polarimetry while the azimuth angle orientation is mainly sensed by azimuth sublooks.

In the first experiment the two dihedrals were $0.7 \mathrm{~m}$ large, while in the second experiment the dihedrals were slightly bigger with dimensions of $1 \mathrm{~m}$.

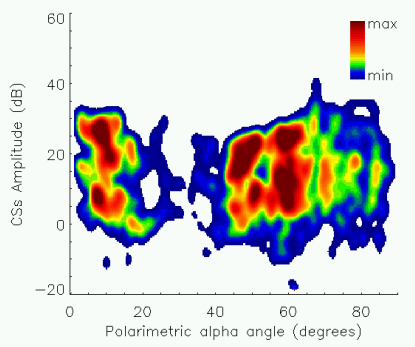

(a) Amplitude $\mathrm{x} \alpha$ angle.

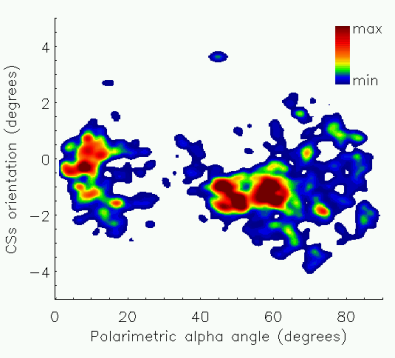

(c) Orientation $\mathrm{x} \alpha$ angle.

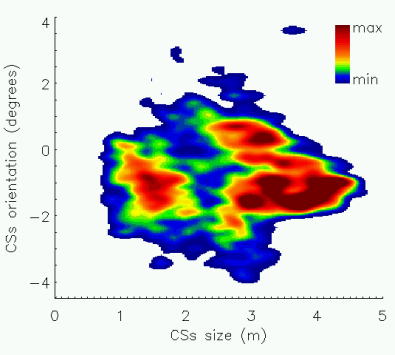

(e) Orientation x Size.

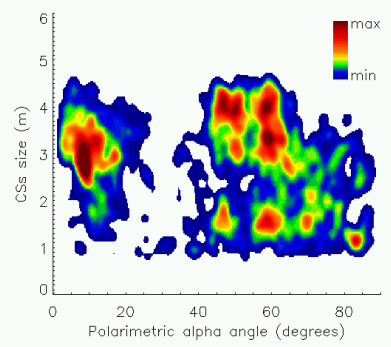

(b) Size $\mathrm{x} \alpha$ angle.

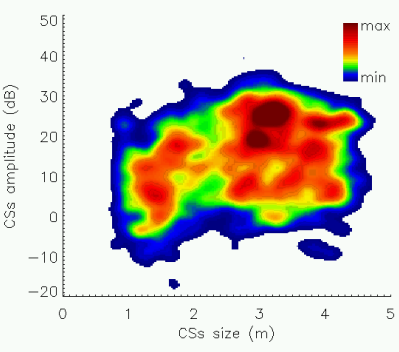

(d) Amplitude x Size.

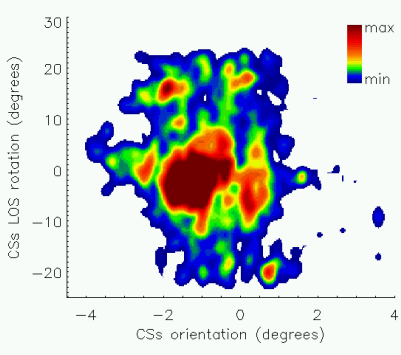

(f) LOS rotation x Orientation.
Fig. 5. Two dimensional histograms relating the polarimetric $\alpha$ angle, the LOS rotation, and the estimated size, orientation and amplitude of CSs.
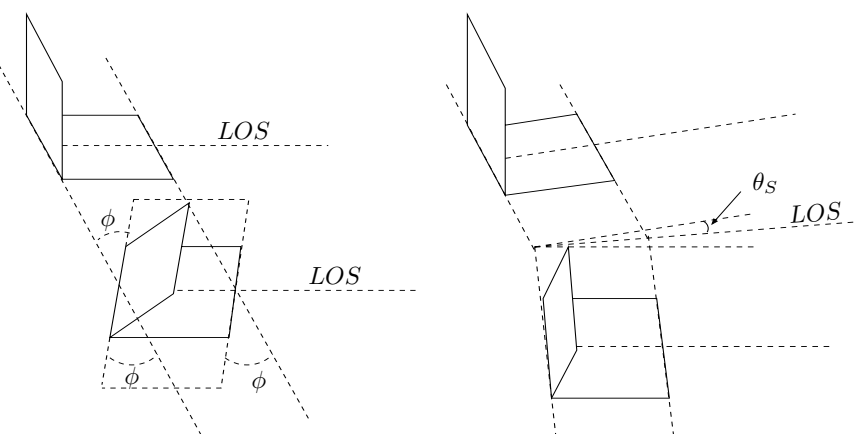

Fig. 6. Dihedrals LOS rotation $\phi$ (left) and azimuth angular orientation $\theta_{S}$ (right). 


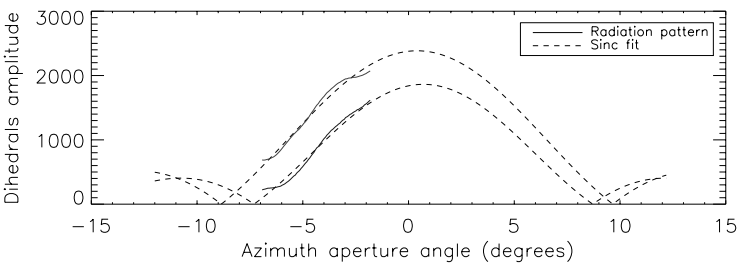

(a) Dihedrals diagram pattern of first experiment.

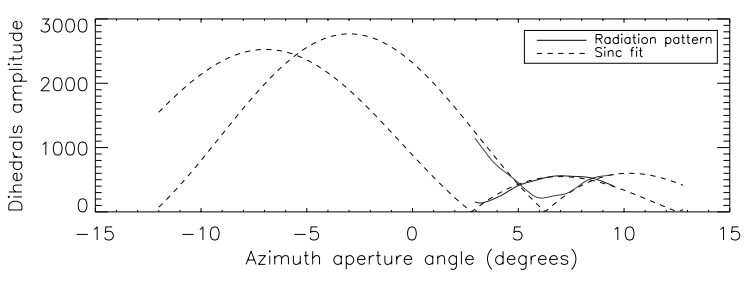

(b) Dihedrals diagram pattern of second experiment.

Fig. 7. Dihedral corner reflectors azimuth radiation patterns.

A. First experiment with 2 dihedrals: $5^{\circ}$ of LOS rotation difference and same azimuth angular orientation

Fig 7(a) shows the estimated azimuth radiation pattern in the $\mathrm{HH}$ image of the two dihedrals for the first experiment. The total azimuth bandwidth has $108.9 \mathrm{~Hz}$, where 76 looks were generated with a sublook bandwidth of $25 \%$ of the full aperture, leading to about $5.1^{\circ}$ of azimuth aspect angle variation. Unfortunately the squint angle was relatively high due to weather conditions $\left(-4.36^{\circ}\right)$, shifting the image spactrum away from the zero degree, to which the dihedrals were oriented.

Due to the high squint and narrow aperture view, only a part of the main-lobe of the dihedrals radiation pattern could be measured. However, the sinc fit indicates that the two patterns have similar widths and are centered approximately at the same place, as expected.

The LOS orientation angle difference of $5^{\circ}$ between the two dihedrals was estimated using the Cameron polarimetric technique [5] and found to be about $4^{\circ}$.

Table I summarises the estimated size, angular orientation, and LOS rotation values of the dihedrals. Considering the narrow azimuth angular aperture, the high squint and that the exact deployment of the dihedrals is limited, the estimation of the dihedral parameters seem quite reasonable.

\section{B. Second experiment with 2 dihedrals: $5^{\circ}$ of azimuth angular orientation difference and same LOS rotation}

In this experiment, both dihedrals were rotated $45^{\circ}$ about the LOS and become visible only in the HV image that has been therefore used to analyse the azimuth radiation patterns. The total azimuth bandwidth was in this case $132.2 \mathrm{~Hz}$, where 66 looks were generated with a sublook bandwidth of $25 \%$ of the full aperture leading to about $6.59^{\circ}$ aspect angle variation.
TABLE I

ESTIMATED DIHEDRALS SIZE, ROTATION AND AZIMUTH ORIENTATION.

\begin{tabular}{|c|c|c|c|c|c|c|c|}
\hline & & $L$ & $L_{\text {est }}$ & $\Delta \theta_{S}$ & $\Delta \theta_{\text {Sest }}$ & $\Delta \phi$ & $\Delta \phi_{\text {est }}$ \\
\hline \hline \multirow{2}{*}{$\begin{array}{c}\text { First } \\
\text { Experim. }\end{array}$} & Dihed. 1 & $0.7 \mathrm{~m}$ & $0.82 \mathrm{~m}$ & $0.0^{\circ}$ & $0.29^{\circ}$ & $5.0^{\circ}$ & $4.02^{\circ}$ \\
\cline { 2 - 7 } & Dihed. 2 & $0.7 \mathrm{~m}$ & $0.71 \mathrm{~m}$ & & & & \\
\hline \hline $\begin{array}{c}\text { Second } \\
\text { Experim. }\end{array}$ & Dihed. 1 & $1.0 \mathrm{~m}$ & $0.71 \mathrm{~m}$ & $5.0^{\circ}$ & $3.97^{\circ}$ & $0.0^{\circ}$ & $0.9^{\circ}$ \\
\cline { 2 - 6 } & Dihed. 2 & $1.0 \mathrm{~m}$ & $0.68 \mathrm{~m}$ & & & & \\
\hline
\end{tabular}

Unfortunately also in this case the squint angle was very high $\left(6.24^{\circ}\right)$, which strongly shifted the azimuth image spectrum away from the zero degree. The sinc fit was nevertheless performed and the results are shown in Fig. 7(b).

Note that, due to the high squint angle, for one dihedral a part of the main-lobe and a part of one side-lobe can be viewed while for the other dihedral only one sidelobe was reconstructable. The estimated shift of the main-lobes is about $4^{\circ}$. Table I summarises the estimated size and angular orientation, as well as the LOS rotation of the dihedrals for this second experiment.

For the estimation of the LOS rotation angles of the two dihedrals in this experiment, a second polarimetric technique (based on the optimum polarimetric states - CTBR class of routines [6]) was used due to the high scatterers LOS rotation $\left(45^{\circ}\right)$. The estimated LOS rotation angles of the dihedrals were $43.7^{\circ}$ and $42.8^{\circ}$.

\section{CONCLUSION}

The potential of using azimuth sublooks to estimate the backscattering radiation pattern of CSs aiming their geometrical characterisation was demonstrated. A simple model for the backscattering radiation of canonical CSs was used and the model parameters were inverted. Using dihedral corner reflectors in controlled experiments, the theory and assumptions were verified and a reasonable agreement was achieved. However, for accurate estimation, a large azimuth bandwidth is essential. For more complex types of CSs, the model of (3) will probably not apply making an extention of the model necessary.

\section{REFERENCES}

[1] R. Z. Schneider, K. P. Papathanassiou, I. Hajnsek, A. Moreira Analysis of Coherent Scatterers over Urban Areas, POLinSAR'2005, Frascati, Italy, January 2005.

[2] R. Z. Schneider, K. P. Papathanassiou, I. Hajnsek, A. Moreira Polarimetric and Interferometric Characterization of Coherent Scatterers in Urban Areas, IEEE Trans. Geoscience and Remote Sensing, vol. 44, No. 4, pp. 971-984, April 2006.

[3] A. Ferretti, D. Perissin, C. Prati and F. Rocca On the Physical Nature of SAR Permanent Scatterers, URSI'2005, Ispra, Italy, April 2005.

[4] S. R. Cloude and E. Pottier An Entropy based Classification Scheme for Land Applications of Polarimetric SAR, IEEE Trans. Geoscience and Remote Sensing, vol. 35, no. 1, pp. 68-78, January 1997.

[5] W. L. Cameron, N.N. Youssef and L.K. Leing Simulated polarimetric signatures of primitive geometrical shapes, IEEE Trans. Geoscience and Remote Sensing, vol. 34, pp. 793-803, May 1996.

[6] W. M. Boerner, W.-L. Yan, A.-Q. Xi and Y. Yamaguchi On the Basic Principles of Radar Polarimetry: the Target Characteristic Polarization State Theory of Kennaugh, Huynen's Polarization Fork Concept, and its Extention to the Partially Polarized Case, Proc. of IEEE, vol. 79, pp. 1538-1550, October 1991. 